\title{
Radiotherapy for inferior vena cava tumor thrombus in patients with hepatocellular carcinoma
}

\author{
Tzu-Hui Pao ${ }^{1 \dagger}$, Wei-Ting Hsueh ${ }^{1 \dagger}$, Wei-Lun Chang ${ }^{2,3}$, Nai-Jung Chiang ${ }^{3,4,5}$, Yih-Jyh Lin ${ }^{6}$, Yi-Sheng Liu ${ }^{7}$ and \\ Forn-Chia Lin ${ }^{1 *}$
}

\begin{abstract}
Background: Hepatocellular carcinoma (HCC) with inferior vena cava (IVC) involvement is a rare disease with poor prognosis. This study aimed to evaluate the outcome of HCC patients receiving radiotherapy (RT) to IVC tumor thrombus.

Methods: A total of 42 consecutive HCC patients treated with RT to IVC tumor thrombus between September 2007 and October 2018 were enrolled. Overall survival (OS), the response of IVC thrombus, prognostic factors and failure pattern were assessed.

Results: The median follow-up time was 4.4 months. The median RT equivalent dose in 2-Gy fractions was 48.75 Gy (range, 3.25-67.10). The objective response rate of IVC thrombus was 47.6\% (95\% confidence interval [CI], 33.3-64. $3 \%)$. The OS rate at 1 year was $30.0 \%$, with a median OS of 6.6 months ( $95 \% \mathrm{Cl}, 3.7-9.5)$ from the start of RT. On multivariate analysis, Child-Pugh class, lymph node metastasis, lung metastasis and objective response of IVC thrombus were independent predictors for OS. Lung was the most common site of first progression in 14 (33.3\%) patients. For 32 patients without lung metastasis before RT, use of systemic treatment concurrent with and/or after RT was associated with a significantly longer lung metastasis-free survival (5.9 vs. 1.5 months, $p=0.0033$ ).

Conclusions: RT is effective for IVC tumor thrombus of HCC with acceptable adverse effects. RT might be a treatment option incorporated into combination therapy for HCC involving IVC.
\end{abstract}

Keywords: Hepatocellular carcinoma, Inferior vena cava thrombus, Radiation therapy

\section{Introduction}

Hepatocellular carcinoma (HCC) is the most common type of liver cancer and the third ranked cause of global cancer mortality [1]. Vascular invasion is a prognostic factor for poor overall survival (OS) in patients with HCC [2, 3]. Compared to portal and hepatic veins, inferior vena cava (IVC) was less frequently involved by HCC. IVC tumor thrombus may flow into heart and lung, leading to pulmonary embolism and lung metastasis. Patients with HCC involving IVC had an increased risk of sudden death and dismal treatment outcome $[4,5]$.

\footnotetext{
* Correspondence: fornchia@mail.ncku.edu.tw

${ }^{\dagger}$ Tzu-Hui Pao and Wei-Ting Hsueh contributed equally to this work.

'Department of Radiation Oncology, National Cheng Kung University

Hospital, College of Medicine, National Cheng Kung University, No.138,

Sheng Li Road, Tainan 70456, Taiwan

Full list of author information is available at the end of the article
}

HCC involving IVC is difficult to treat and a standard therapy has not been established. Surgery, transarterial chemoembolization (TACE) and systemic treatment were adopted in the management of these cases. The use of radiotherapy (RT) remains controversial. Some retrospective studies have suggested that RT is a feasible and safe option to palliate HCC with IVC invasion with pooled 1-year OS rate of 53.6\%, response rate of 59.2\% and possible severe complication rate of $1.2 \%$ [6]. However, the data regarding lung metastasis and pulmonary embolism after RT for these patients was limited.

In the current study, we retrospectively evaluated the clinical outcomes and prognostic factors in HCC patients receiving RT to IVC tumor thrombus. In addition, the information of lung metastasis and pulmonary embolism before and after RT was reported.

(c) The Author(s). 2019 Open Access This article is distributed under the terms of the Creative Commons Attribution 4.0 International License (http://creativecommons.org/licenses/by/4.0/), which permits unrestricted use, distribution, and reproduction in any medium, provided you give appropriate credit to the original author(s) and the source, provide a link to the Creative Commons license, and indicate if changes were made. The Creative Commons Public Domain Dedication waiver (http://creativecommons.org/publicdomain/zero/1.0/) applies to the data made available in this article, unless otherwise stated. 


\section{Methods}

\section{Patients}

This study enrolled 42 consecutive $\mathrm{HCC}$ patients receiving RT to IVC tumor thrombus at our hospital from September 2007 to October 2018. The demographic and clinical features, treatment modalities and outcomes of these patients were gathered from a review of the medical records. HCC was diagnosed on the basis of histological examination or the image criteria of the American Association for the Study of Liver Diseases guideline [7]. The IVC tumor thrombus was diagnosed by characteristic finding of computed tomography (CT) or magnetic resonance imaging (MRI). All patients had pretreatment assessment consisted of a history and physical examination, hematology, biochemistry, Hepatitis $\mathrm{B} / \mathrm{C}$ panel and chest radiographs.

\section{Radiotherapy}

Radiotherapy was delivered by using a linear accelerator with intensity-modulated radiotherapy (IMRT) in 35 patients and three dimensional conformal radiation therapy (3DCRT) in seven patients. All patients were immobilized by customized devices in the supine position with both arms raised above the head. The simulation CT scan was acquired at $5 \mathrm{~mm}$ slice thickness and transferred for treatment planning system to determine the radiation volume and dose distribution. RT was planned to target IVC and right atrium thrombus. Synchronously, hepatic or portal vein thrombus was also irradiated in 26 patients and intrahepatic tumor in seven patients according to the suggestion of our institutional multidisciplinary HCC team. The gross tumor volume (GTV) was defined as the hypodense filling defect area for venous thrombus and the hyperdense area for intrahepatic tumors. The clinical target volume (CTV) was defined as GTV plus a $0.5-1 \mathrm{~cm}$ margin along the vein for thrombi and in all directions for intrahepatic tumors. For cases with tumor thrombus in the right atrium, we included the whole right atrium in the CTV. The planning target volume (PTV) was determined by adding a 0.5-1 cm margin to the CTV for uncertainties in treatment delivery. A daily dose of 2-3 Gy was delivered to the PTV using 6- or 10- MV X-rays at five fractions per week. The RT dose was converted to equivalent dose in $2-G y$ fractions for $\alpha / \beta=10\left(E_{10 / 2}\right)$. Other therapies for HCC given within 4 weeks before or after RT was defined as concurrent treatments.

\section{Response assessments and follow-up}

CT or MRI scans were performed at 1 to 3 months after the completion of RT and then every 3 to 6 months thereafter. The response of the IVC tumor thrombus was assessed according to the World Health Organization criteria [8]. The product of the two greatest perpendicular diameters of the IVC tumor thrombus was calculated and compared with the baseline value. Complete disappearance of IVC tumor thrombus was defined as complete response (CR), decrease of $\geq 50 \%$ in IVC thrombus size as partial response (PR), decrease of $<50 \%$ in IVC tumor thrombus or increase of $<25 \%$ as stable disease (SD), and increase of $\geq 25 \%$ as progressive disease (PD). The objective response included CR and PR. Adverse effects were graded according to the Common Terminology Criteria for Adverse Events (CTCAE; version 3.0).

\section{Statistical methods}

The data cutoff date was October 10, 2018. The survival curves were estimated using the Kaplan-Meier method and compared statistically using the log-rank test. OS was calculated from the start of RT to the date of death. For patients without lung metastasis before RT, lung metastasis-free survival was measured from the start of $\mathrm{RT}$ to the date of death or the development of lung metastasis. Univariate and multivariate Cox proportional hazards analyses were performed to check factors associated with $\mathrm{OS}$ and lung metastasis-free survival. A $p$ value of $<0.05$ was considered statistically significant. Data analysis was performed with SPSS version 22.0 software and $\mathrm{R}$ version 3.5.1 for Windows.

\section{Results}

\section{Patient characteristics}

The demographic and clinical characteristics of 42 patients at baseline are summarized in Table 1. The cohort included 29 men (69\%) and 13 women (31\%). The majority of patients (90.5\%) had hepatitis B and/or C. Eight (19.0\%) patients had lymph node (LN) metastasis and ten $(23.8 \%)$ patients had lung metastasis. In addition to IVC, thrombus was also noted in right atrium in 11 (26.2\%), portal vein in 16 (38.1\%), and hepatic vein in 15 (35.7\%) patients.

\section{Treatment characteristics}

All treatment modalities are summarized in Table 2. Before RT to IVC thrombus, ten patients were treated by surgery, 19 by TACE/TAE (transarterial embolization), and 11 by radiofrequency ablation (RFA) or percutaneous ethanol injection (PEI). Thirty-six patients completed the RT treatment while RT was terminated earlier in six patients ( 3 due to terminal liver disease, 2 pulmonary embolism and 1 respiratory failure). The median RT EQD 10/2 were 48.75 Gy (range, 3.25-67.10). Within 4 weeks before or after RT, 13 patients underwent TAE/ TACE or RFA/PEI for their intrahepatic tumors. Furthermore, twelve (28.6\%), 24 (57.1\%) and 21 (50.0\%) patients received individualized systemic treatment before, during and after RT, respectively (Table 3). Sorafenib 
Table 1 Demographic and Clinical Characteristic of Patients at Baseline

\begin{tabular}{|c|c|}
\hline Characteristic & No. of patients (\%) \\
\hline \multicolumn{2}{|l|}{ Age (years) } \\
\hline Median & 63 \\
\hline Range & $40-92$ \\
\hline \multicolumn{2}{|l|}{ Gender } \\
\hline Male & $29(69.0)$ \\
\hline Female & $13(31.0)$ \\
\hline \multicolumn{2}{|c|}{ ECOG performance status } \\
\hline 0 & $4(9.5)$ \\
\hline 1 & $23(54.8)$ \\
\hline 2 & $14(33.3)$ \\
\hline 3 & $1(2.4)$ \\
\hline \multicolumn{2}{|l|}{ Child-Pugh class } \\
\hline A & $25(59.5)$ \\
\hline B & $15(35.7)$ \\
\hline C & $2(4.8)$ \\
\hline \multicolumn{2}{|c|}{ Etiology of chronic liver disease } \\
\hline HBV & $24(57.1)$ \\
\hline $\mathrm{HCV}$ & $11(26.2)$ \\
\hline $\mathrm{HBV} \& \mathrm{HCV}$ & $3(7.1)$ \\
\hline Others & $4(9.5)$ \\
\hline \multicolumn{2}{|c|}{ Thrombus site besides IVC ${ }^{a}$} \\
\hline Right atrium & $11(26.2)$ \\
\hline Portal vein & $16(38.1)$ \\
\hline Hepatic vein & $15(35.7)$ \\
\hline \multicolumn{2}{|l|}{ LN metastasis } \\
\hline Absent & $34(81.0)$ \\
\hline Present & $8(19.0)$ \\
\hline \multicolumn{2}{|l|}{ Lung metastasis } \\
\hline Absent & $32(76.2)$ \\
\hline Present & $10(23.8)$ \\
\hline
\end{tabular}

Abbreviations: ECOG Eastern Cooperative Oncology Group, $H B V$ hepatitis B virus, $H C V$ hepatitis $C$ virus, IVC inferior vena cava, $L N$ lymph node

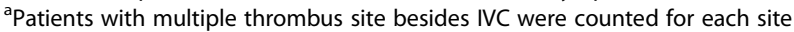

was the most commonly used systemic anticancer drug, with a median treatment duration of 3.3 months in 31 patients.

\section{IVC response and survival outcomes}

The median follow-up time was 4.4 months (range, 0.26-55.89) in the whole cohort. Fifteen patients died before CT or MRI for the response evaluation. Among the 27 evaluable patients, CR, PR and SD were achieved in four, 16 and seven patients, respectively. For the entire cohort, the objective response rate was $47.6 \%$. The 1-year OS rate was $30.0 \%$, with a median survival of 6.6 months (95\% CI 3.7-9.5; Fig. 1). The OS was not
Table 2 Treatment Characteristics

\begin{tabular}{|c|c|}
\hline Treatment & No. of patients (\%) \\
\hline \multicolumn{2}{|l|}{ Previous treatment ${ }^{\mathrm{a}}$} \\
\hline None & $15(35.7)$ \\
\hline Operation & $10(23.8)$ \\
\hline TACE/TAE & $19(45.2)$ \\
\hline RFA/PEI & $11(26.2)$ \\
\hline Systemic treatment & $12(28.6)$ \\
\hline \multicolumn{2}{|c|}{ Treatment concurrent with $\mathrm{RT}^{\mathrm{b}}$} \\
\hline None & $13(31.0)$ \\
\hline Operation & $0(0.0)$ \\
\hline TACE/TAE & $12(28.6)$ \\
\hline RFA/PEI & $1(2.4)$ \\
\hline Systemic treatment & $24(57.1)$ \\
\hline \multicolumn{2}{|l|}{ Treatment after $\mathrm{RT}^{\mathrm{C}}$} \\
\hline None & $20(47.6)$ \\
\hline Operation & $4(9.5)$ \\
\hline TACE/TAE & $6(14.3)$ \\
\hline RFA/PEI & $1(2.4)$ \\
\hline Systemic treatment & $21(50.0)$ \\
\hline \multicolumn{2}{|l|}{ RT technique } \\
\hline 3DCRT & $7(16.7)$ \\
\hline IMRT & $35(83.3)$ \\
\hline \multicolumn{2}{|l|}{ RT dose $\left(E Q D_{10 / 2}, G y\right)^{d}$} \\
\hline Median (range) & $48.75(3.25-67.10)$ \\
\hline$<50$ & $22(52.4)$ \\
\hline$\geq 50$ & $20(47.6)$ \\
\hline
\end{tabular}

Abbreviations: $R T$ radiotherapy, TACE transarterial chemoembolization, TAE transarterial embolization, $R F A$ radiofrequency ablation, $P E I$ percutaneous ethanol injection, 3DCRT three-dimensional conformal radiotherapy, IMRT intensity modulation radiation therapy

aTreatment given beyond 4 weeks before RT began

${ }^{\mathrm{b}}$ Treatment given within 4 weeks before RT began or 4 weeks after

RT completed

'Treatment given beyond 4 weeks after RT completed

${ }^{\mathrm{d}}$ Equivalent dose in $2 \mathrm{~Gy}$ fractions, $\alpha / \beta=10$

Table 3 Systemic Therapy

\begin{tabular}{llll}
\hline & \multicolumn{3}{l}{ No. of patients (\%) } \\
\cline { 2 - 4 } Systemic therapy & Pre-R/T & During RT & Post-R/T \\
\hline Total & $12(28.6)$ & $24(57.1)$ & $21(50.0)$ \\
Sorafenib & $12(28.6)$ & $17(40.5)$ & $16(38.1)$ \\
Nivolumab & $1(2.4)$ & $2(4.8)$ & $2(4.8)$ \\
Thalidomide & $0(0.0)$ & $4(9.5)$ & $1(2.4)$ \\
Tegafur/uracil & $0(0.0)$ & $1(2.4)$ & $0(0.0)$ \\
Ramucirumab & $0(0.0)$ & $0(0.0)$ & $3(7.1)$ \\
Everolimus & $0(0.0)$ & $0(0.0)$ & $1(2.4)$ \\
Pegargiminase & $0(0.0)$ & $0(0.0)$ & $1(2.4)$ \\
\hline
\end{tabular}


associated with RT techniques (2.1 and 7.1 months in 3DCRT and IMRT group, respectively, $p=0.33$ ). On the hand, the median OS was significantly longer for patients with Child-Pugh class (CPC) A (11.5 vs. 1.8 months, $p<0.0001$; Fig. 2a), without LN metastasis (7.4 vs. 1.9 months, $p=0.002$; Fig. $2 \mathrm{~b}$ ), without lung metastasis (7.1 vs. 1.9 months, $p=0.044$; Fig. $2 \mathrm{c}$ ), and RT $\mathrm{EQD}_{10 / 2} \geq 50$ Gy (8.0 vs. 3.6 months, $p=0.011$; Fig. $2 \mathrm{~d}$ ). Furthermore, the objective response of IVC was associated with a longer median OS in the entire cohort (12.4 vs. 2.1 months, $p<0.0001$; Fig. 3a). Among the 27 evaluable patients, there was a trend toward better OS in the objective responder of IVC ( $p=0.066$; Fig. $3 \mathrm{~b})$. Multivariate analysis confirmed that $\mathrm{CPC}$, LN metastasis, lung metastasis and objective response of IVC thrombus were independent predictors for OS (Table 4).

\section{Lung metastasis and pulmonary embolism}

Ten (23.8\%) patients had lung metastasis before RT to IVC thrombus. During post-RT follow-up, lung metastasis was identified in additional 20 patients (Fig. 4a). Among the 32 patients without lung metastasis before RT, use of systemic therapy concurrently with and/or after RT was associated with a longer median lung metastasis-free survival (5.9 vs. 2.1 months, $p=0.0033$; Fig. 5a). By multivariate analysis, objective response of IVC thrombus was the only independent predictor for lung metastasis-free survival (Table 5). Patients with objective response of IVC thrombus had a longer median lung metastasis-free survival (6.5 vs. 1.9 months, $p=$ 0.002; Fig. 5b). Furthermore, six (14.3\%) patients had pulmonary embolism before RT. During post-RT follow-up, resolution of pulmonary embolism was noted

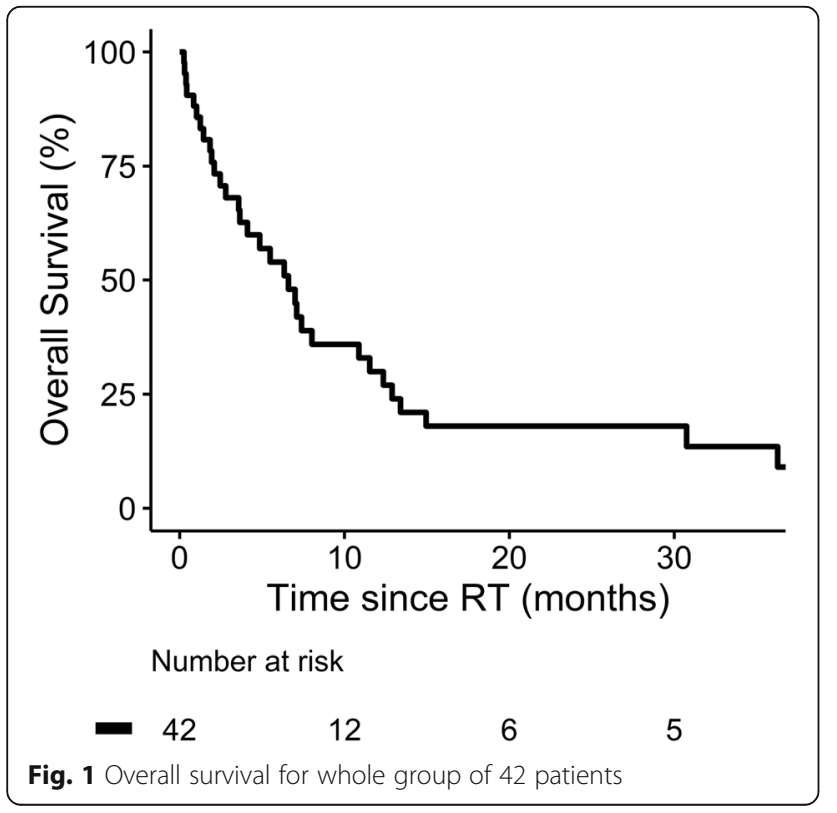

in two patients who received anticoagulant and systemic treatment (1 with sorafenib followed by ramucirumab, and 1 everolimus followed by sorafenib). On the other hand, one of 36 patients without pulmonary embolism before RT had pulmonary embolism during post-RT follow-up (Fig. 4b).

\section{Patterns of failure}

At the data cutoff, $33(78.6 \%)$ patients died. The site of first progression was shown in Table 6. The most common site was lung in 14 (33.3\%) patients. Only three (7.1\%) patients had their first progression in the IVC thrombus. The most common causes of death were liver failure due to tumor progression or hepatic decompensation in $11(33.3 \%)$ and lung metastasis in seven (21.2\%) patients.

\section{Toxicity}

The most common acute toxicities were fatigue in 13 (31\%), dysphagia in four (9.5\%), radiation dermatitis in three (7.1\%) and cough in one (2.4\%) patient. These toxicities were grade 1 or 2 . In addition, elevation of Child-Pugh score $\geq 2$ were observed in nine (21.4\%) patients.

\section{Discussion}

HCC involving IVC is a challenging clinical condition. The optimal treatment remains undetermined. Previous studies suggested the potential therapeutic role of RT in these patients [6, 9-16]. In the present study, 42 HCC patients were treated with RT to IVC thrombus (7 with 3-DCRT and 35 IMRT). RT was earlier stopped in six patients ( 3 due to terminal liver disease, 2 pulmonary embolism and 1 respiratory failure). The remaining 36 patients completed RT with median $\mathrm{EQD}_{10 / 2}$ of $50 \mathrm{~Gy}$. For all 42 study patients, the median OS was 6.6 months and 1-year OS rate was $30.0 \%$. This result was similar to the experience of two Asian institutions [10,11] but compared unfavorably with the pooled data from prior eight studies (1-year OS rate of 53.6\%) [6]. We next examined the possible factors contributing to this dismal outcome. A multivariable analysis of patient characteristics demonstrated that $\mathrm{CPC} \mathrm{B} / \mathrm{C}$, $\mathrm{LN}$ metastasis, lung metastasis and the absence of objective response of IVC thrombus were associated with poorer OS.

LN metastasis and liver cirrhosis are key prognostic determinants of HCC patients. In our cohort focusing on HCC involving IVC, eight patients with LN metastasis received individualized systemic treatment for the involved LN. These patients had a significantly inferior OS of 1.9 months when compared to those without LN metastasis. It is interesting to note that $\mathrm{LN}$ metastasis was still an independent predictor of survival in so advanced stage HCC. This result was in line with prior studies [9, 

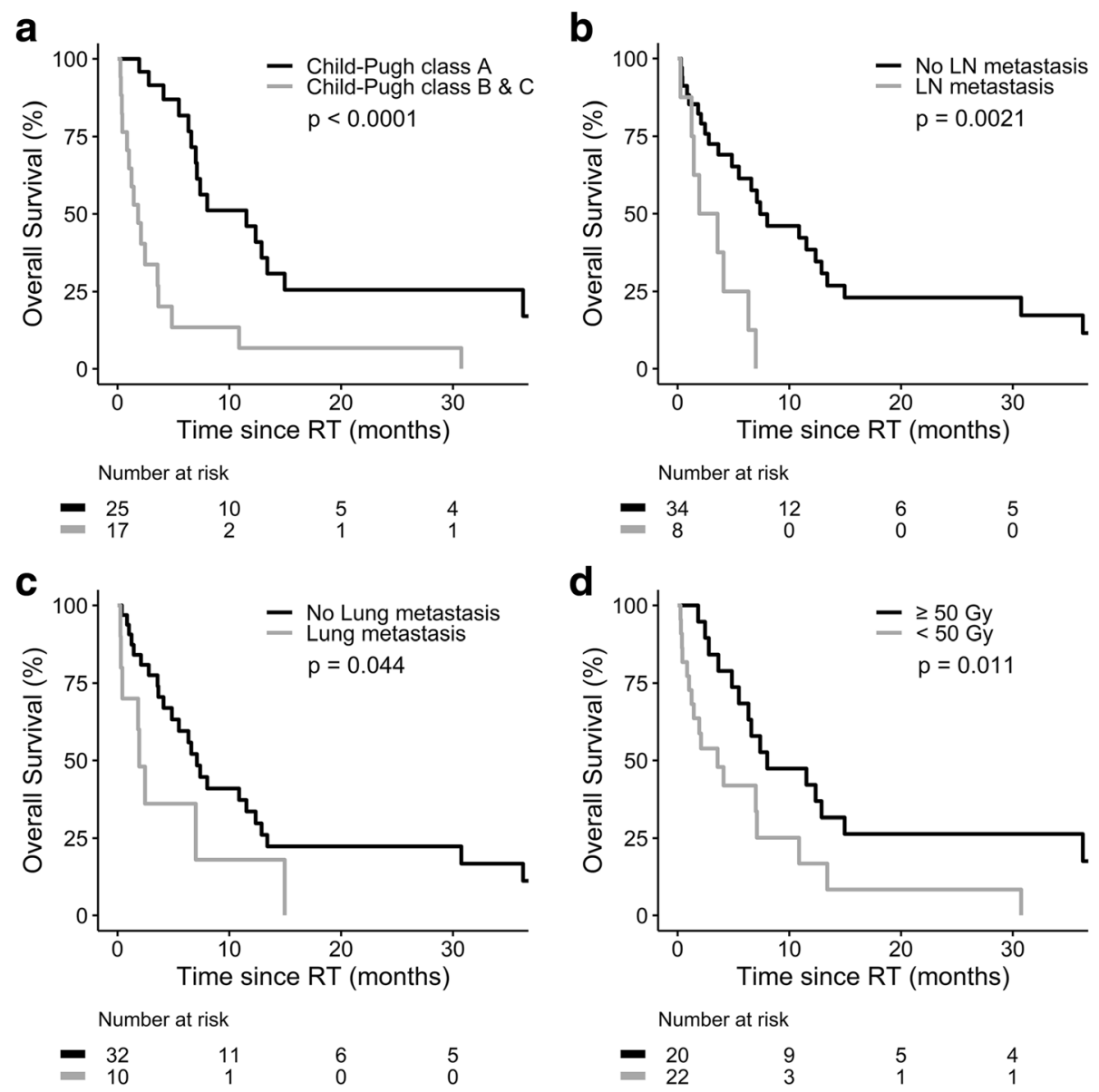

Fig. 2 Overall survival by (a) Child-Pugh class, (b) lymph node (LN) metastasis, (c) lung metastasis before radiotherapy, and (d) radiotherapy dose $\left(E Q D_{10 / 2}\right.$, equivalent dose in 2-Gy fractions, $\left.a / \beta=10\right)$
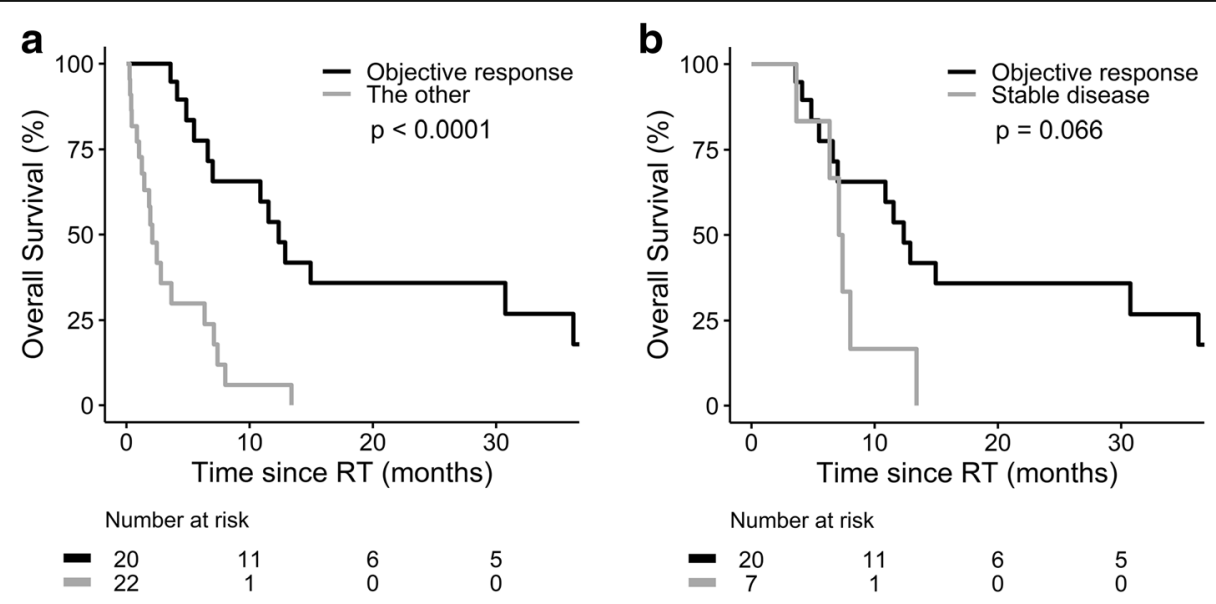

Fig. 3 Overall survival by IVC response in (a) whole 42 patients, and (b) 27 evaluable patients 
Table 4 Univariate and Multivariate Analysis of Covariables Associated with Overall Survival

\begin{tabular}{|c|c|c|c|c|}
\hline \multirow[t]{2}{*}{ Variable } & \multicolumn{2}{|l|}{ Univariate analysis } & \multicolumn{2}{|l|}{ Multivariate analysis } \\
\hline & $\mathrm{HR}(95 \% \mathrm{Cl})$ & $P$ value & $\mathrm{HR}(95 \% \mathrm{Cl})$ & $P$ value \\
\hline Age (<65 vs. $\geq 65$ years) & $0.709(0.350-1.434)$ & 0.338 & & \\
\hline Gender (female vs. male) & $0.748(0.502-1.114)$ & 0.153 & & \\
\hline ECOG performance status (2-3 vs. $0-1$ ) & $1.656(1.138-2.410)$ & 0.008 & $1.175(0.365-3.776)$ & 0.787 \\
\hline Child-Pugh class (B\&C vs. A) & $2.123(1.472-3.062)$ & 0.000 & $2.411(1.073-5.414)$ & 0.033 \\
\hline Right atrium invasion (yes vs. no) & $1.330(0.905-1.952)$ & 0.146 & & \\
\hline Portal vein invasion (yes vs. no) & $1.961(1.343-2.864)$ & 0.000 & $1.086(0.662-1.782)$ & 0.743 \\
\hline Hepatic vein invasion (yes vs. no) & $0.970(0.662-1.422)$ & 0.876 & & \\
\hline LN metastasis (yes vs. no) & $1.926(1.232-3.010)$ & 0.004 & $2.118(1.171-3.831)$ & 0.013 \\
\hline Lung metastasis (yes vs. no) & $1.509(1.000-2.276)$ & 0.050 & $1.751(1.064-2.883)$ & 0.028 \\
\hline Systemic treatment ${ }^{a}$ (without vs. with) & $2.430(1.625-3.634)$ & 0.000 & $1.346(0.680-2.667)$ & 0.394 \\
\hline RT dose $\left(E D_{10 / 2}, G y\right)^{b}(<50$ vs. $\geq 50)$ & $1.583(1.100-2.280)$ & 0.013 & $1.050(0.669-1.649)$ & 0.832 \\
\hline Objective response of $\mathrm{IVC}^{\mathrm{C}}$ (no vs. yes) & $2.326(1.552-3.488)$ & 0.000 & $2.604(1.544-4.391)$ & 0.000 \\
\hline
\end{tabular}

Abbreviations: ECOG Eastern Cooperative Oncology Group, LN lymph node, RT radiotherapy, IVC inferior vena cava

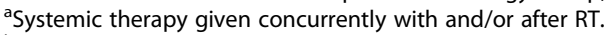

${ }^{b}$ Equivalent dose in 2 Gy fractions, $a / \beta=10$

'Objective response: complete and partial responses

13]. Moreover, CPC is widely used for the clinical assessment of liver cirrhosis and liver function. In our study, patients with CPC A had a longer median OS time than those with CPC B/C (11.5 vs 1.8 months). Similar findings were disclosed in prior reports which showed a longer median OS of 12.2 months in patients with CPC A than 6.1 months in CPC B [9], and confirmed CPC A was an independent predictor for better OS [12]. Collectively, the results of our and previous studies indicated that the residual liver function was an important clinical factor in these patients who commonly have concomitant intra-/extra-hepatic lesions needing further therapies.

Lung is a common site of extrahepatic dissemination in HCC. Of 42 patients in the current study, ten patients had lung metastasis before RT. The presence of lung metastasis before RT was correlated with poor OS. To our knowledge, this is the first study to show lung metastasis before RT was an independent predictor for OS in HCC patients receiving RT to IVC tumor thrombus. Furthermore, lung metastasis was found in additional 20 patients during post-RT follow-up. From the present retrospective analysis, it is difficult to attribute these newly developed lung metastases to thrombus dislodgment during RT or the natural history of cancer. On the other hand, we showed that objective response of IVC thrombus was an independent predictor for lung metastasis-free survival. Systemic therapy given concurrently with and/or after RT was associated with a longer lung metastasis-free survival in 32 patients who did not a

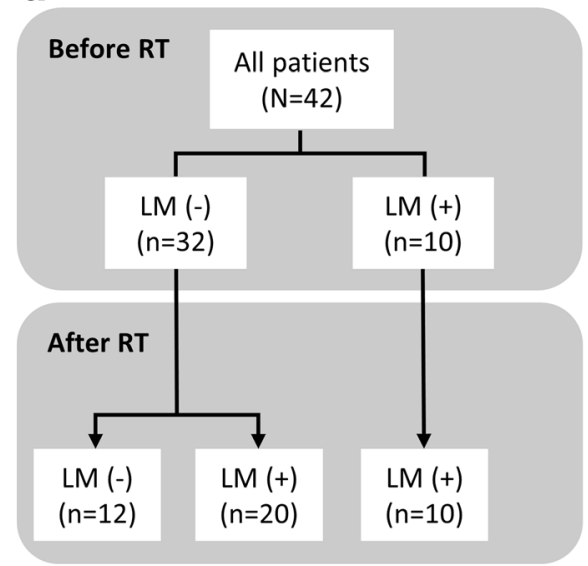

b

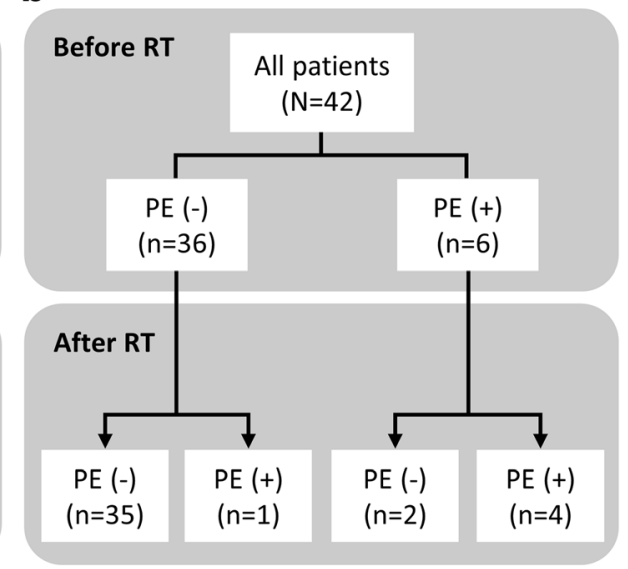

Fig. 4 Status of (a) lung metastasis (LM), and (b) pulmonary embolism (PE) before and after RT 

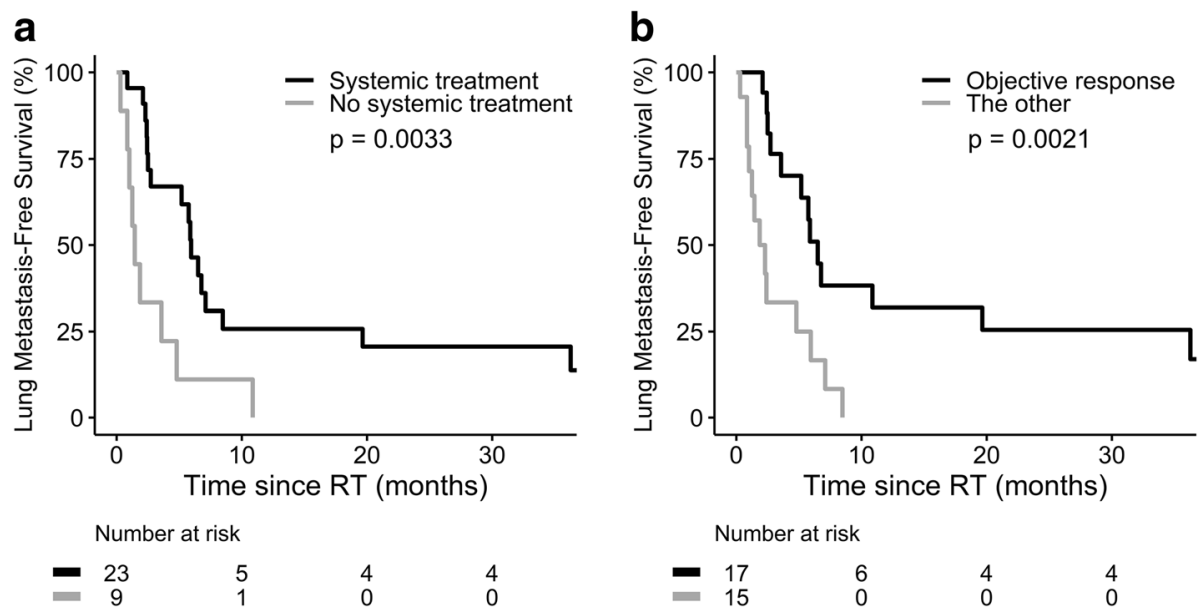

Fig. 5 Lung metastasis-free survival by (a) use of systemic treatment concurrent with and/or after RT, and (b) IVC response in 32 patients without lung metastasis before RT

have lung metastasis before RT. This finding suggested the potential role of systemic therapy on reducing lung metastasis after RT to IVC tumor thrombus.

Advances in radiation technology made it possible to deliver higher RT dose to the tumor without causing severe complications. In the present study using modern RT for IVC tumor thrombus, patients receiving RT $\mathrm{EQD}_{10 / 2}$ higher than $50 \mathrm{~Gy}$ had a longer median OS time than those of lower dose. Moreover, objective response of IVC thrombus to RT was an independent predictor for better OS. This finding was consistent with the result of the prior study [13]. On the other hand, RT-related adverse effects were major concerns in these HCC patients with advanced stage and limited survival. Pulmonary embolism was suspected to be a complication due to thrombus dislodgment during RT in one study [11]. In the current cohort, pulmonary embolism was identified in six patients before RT. Among the remaining 36 patients, only one patient had pulmonary embolism during post-RT follow-up. This finding suggested pulmonary embolism was more likely a common natural consequence of IVC thrombus than complication of RT. However, we cannot exclude the possibility that this newly developed pulmonary embolism of our cohort was due to RT-induced thrombus dislodgment. Aside from this possible adverse event of pulmonary embolism, no other severe side effect was noted in the present study. This rarity of severe complications might be due to underestimation in our retrospective review for patients with short survival time. But on the positive side, the low

Table 5 Univariate and Multivariate Analysis of Covariables Associated with Lung Metastasis-Free Survival

\begin{tabular}{|c|c|c|c|c|}
\hline \multirow[t]{2}{*}{ Variable } & \multicolumn{2}{|l|}{ Univariate analysis } & \multicolumn{2}{|l|}{ Multivariate analysis } \\
\hline & $\mathrm{HR}(95 \% \mathrm{Cl})$ & $P$ value & $\mathrm{HR}(95 \% \mathrm{Cl})$ & $P$ value \\
\hline Age (<65 vs. $\geq 65$ years) & $0.745(0.344-1.617)$ & 0.457 & & \\
\hline Gender (female vs. male) & $0.664(0.284-1.552)$ & 0.344 & & \\
\hline ECOG performance status ( $2-3$ vs. $0-1$ ) & 4.069 (1.665-9.943) & 0.002 & $3.337(0.895-12.440)$ & 0.073 \\
\hline Child-Pugh class (B\&C vs. A) & $3.336(1.475-7.543)$ & 0.004 & $2.847(0.867-9.354)$ & 0.085 \\
\hline Right atrium invasion (yes vs. no) & $2.134(0.906-5.028)$ & 0.083 & & \\
\hline Portal vein invasion (yes vs. no) & $2.614(1.109-6.162)$ & 0.028 & $1.149(0.406-3.250)$ & 0.794 \\
\hline Hepatic vein invasion (yes vs. no) & $0.973(0.436-2.172)$ & 0.947 & & \\
\hline LN metastasis (yes vs. no) & $4.360(1.415-13.429)$ & 0.010 & $1.016(0.248-4.171)$ & 0.982 \\
\hline Systemic treatment ${ }^{a}$ (without vs. with) & $3.263(1.332-7.988)$ & 0.010 & $2.463(0.919-6.600)$ & 0.073 \\
\hline RT dose $\left(\mathrm{EQD}_{10 / 2}, \mathrm{~Gy}\right)^{\mathrm{b}}(<50$ vs. $\geq 50)$ & $2.171(0.962-4.896)$ & 0.062 & & \\
\hline Objective response of IVC (no vs. yes) & $3.471(1.498-8.042)$ & 0.004 & $5.732(1.906-17.235)$ & 0.002 \\
\hline
\end{tabular}

Abbreviations: ECOG Eastern Cooperative Oncology Group, LN lymph node, $R T$ radiotherapy, IVC inferior vena cava

${ }^{\text {a }}$ Systemic therapy given concurrently with and/or after RT.

${ }^{b}$ Equivalent dose in $2 \mathrm{~Gy}$ fractions, $\alpha / \beta=10$

'Objective response: complete and partial responses 
Table 6 Site of First Progression and Cause of Death

\begin{tabular}{ll}
\hline Disease & No. of patients (\%) \\
\hline Site of first progression & $3(7.2)$ \\
IVC thrombus & $9(21.5)$ \\
Liver & $21(50.0)$ \\
Distant metastasis & $14(33.3)$ \\
$\quad$ Lung & $3(7.1)$ \\
Brain & $2(4.8)$ \\
Bone & $2(4.8)$ \\
$\quad$ Others & \\
Cause of death & $11(33.3)$ \\
Liver failure & $7(21.2)$ \\
Lung metastasis & $2(6.1)$ \\
Pulmonary embolism & $2(6.1)$ \\
Variceal bleeding & $2(6.1)$ \\
Duodenal ulcer bleeding & $2(6.1)$ \\
Pneumonia & $2(6.1)$ \\
SBP & $1(3.0)$ \\
Brain metastasis & $1(3.0)$ \\
Acute renal failure & $1(3.0)$ \\
CVA & $2(6.1)$ \\
Unknown &
\end{tabular}

Abbreviations: SBP spontaneous bacterial peritonitis, CVA cerebrovascular accident

complication rate may reflect the advantage of 3DCRT and IMRT which delivered relatively low RT dose to normal organs.

Our study has several limitations. First, the study is retrospective and carries with it all of the biases inherent in such an analysis. Specifically, treatment related morbidity might be underestimated due to insufficient information in the medical record. Second, the number of enrolled patients was small. It is possible to miss significant relationships from the data. Finally, our study investigated patients treated between 2007 and 2018 . Changes in therapies for HCC and supportive management over time may affect patient outcome and contribute to study bias.

\section{Conclusions}

In conclusion, this study showed RT was a feasible and safe treatment option for IVC tumor thrombus in HCC patients. Detailed information relating to pulmonary embolism and lung metastasis was provided in the present study. Pulmonary embolism due to thrombus dislodgment during RT rarely occurred. Use of systemic therapy given concurrently with and/or after RT was associated with a longer lung metastasis-free survival. Based on our results, RT might be a treatment option incorporated into combination therapy for HCC involving IVC.

\section{Abbreviations}

3DCRT: Three dimensional conformal radiation therapy; Cl: Confidence interval; CPC: Child-Pugh class; CR: Complete response; CT: Computed tomography; CTV: Clinical target volume; ECOG: Eastern Cooperative Oncology Group; EQD 10/2: Equivalent dose in 2-Gy fractions, $\alpha / \beta=10$; GTV: Gross tumor volume; Gy: Gray; HCC: Hepatocellular carcinoma; IMRT: Intensity-modulated radiotherapy; IVC: Inferior vena cava; LN: Lymph node; MRI: Magnetic resonance imaging; OS: Overall survival; PD: Progressive disease; PE: Pulmonary embolism; PEl: Percutaneous ethanol injection; PR: Partial response; PTV: Planning target volume; RFA: Radiofrequency ablation; RT: Radiotherapy; SD: Stable disease; TACE: Transarterial chemoembolization; TAE: Transarterial embolization

\section{Acknowledgements}

Not applicable.

\section{Funding \\ This work was supported by the Ministry of Science and Technology of Taiwan [MOST 105-2314-B-006-045-MY2 to FCL] and National Cheng Kung University Hospital of Taiwan (NCKUH-10803007 to WTH). The funding sources played no role in the design of the study and collection, analysis, and interpretation of data nor in the writing of the manuscript.}

Availability of data and materials

The datasets used and/or analyzed during the current study are available from the corresponding author on reasonable request.

\section{Authors' contributions}

THP, WLC, NJC, YJL, and FCL participated in the design. WTH, THP, NJC, YSL, and FCL participated in data collection. WTH, THP, WLC, and FCL participated in data analysis. All authors participated in data interpretation, drafting, and finalizing the report. All authors read and approved the final manuscript.

Ethics approval and consent to participate

This study was approved by the Institutional Review Board of National Cheng Kung University Hospital (reference number, A-ER-107-349). The informed consent was waived because of the retrospective nature of the study.

Consent for publication

Not applicable.

\section{Competing interests}

The authors declare that they have no competing interests.

\section{Publisher's Note}

Springer Nature remains neutral with regard to jurisdictional claims in published maps and institutional affiliations.

\section{Author details}

${ }^{1}$ Department of Radiation Oncology, National Cheng Kung University Hospital, College of Medicine, National Cheng Kung University, No.138, Sheng Li Road, Tainan 70456, Taiwan. ²Department of Internal Medicine, National Cheng Kung University Hospital, College of Medicine, National Cheng Kung University, Tainan, Taiwan. ${ }^{3}$ Institute of Clinical Medicine, College of Medicine, National Cheng Kung University, Tainan, Taiwan. ${ }^{4}$ National Institute of Cancer Research, National Health Research Institutes, Tainan, Taiwan. ${ }^{5}$ Division of Hematology/Oncology, Department of Internal Medicine, National Cheng Kung University Hospital, Tainan, Taiwan. ${ }^{6}$ Division of Transplant surgery, Department of Surgery, National Cheng Kung University Hospital, College of Medicine, National Cheng Kung University, Tainan, Taiwan. ${ }^{7}$ Department of Diagnostic Radiology, National Cheng Kung University Hospital, College of Medicine, National Cheng Kung University, Tainan, Taiwan. 
Received: 17 December 2018 Accepted: 29 April 2019

Published online: 10 June 2019

\section{References}

1. Ferlay J, Soerjomataram I, Dikshit R, Eser S, Mathers C, Rebelo M, et al. Cancer incidence and mortality worldwide: sources, methods and major patterns in GLOBOCAN 2012. Int J Cancer. 2015;136(5):E359-86.

2. Fong $Y$, Sun $R L$, Jarnagin $W$, Blumgart LH. An analysis of 412 cases of hepatocellular carcinoma at a Western center. Ann Surg. 1999;229(6):790-9 discussion 99-800.

3. Park KW, Park JW, Choi Jl, Kim TH, Kim SH, Park HS, et al. Survival analysis of 904 patients with hepatocellular carcinoma in a hepatitis B virus-endemic area. J Gastroenterol Hepatol. 2008;23(3):467-73.

4. Nagasue N, Yukaya H, Hamada T, Hirose S, Kanashima R, Inokuchi K. The natural history of hepatocellular carcinoma. A study of 100 untreated cases. Cancer. 1984;54(7):1461-5.

5. Llovet JM, Bustamante J, Castells A, Vilana R, Ayuso Mdel C, Sala M, et al. Natural history of untreated nonsurgical hepatocellular carcinoma: rationale for the design and evaluation of therapeutic trials. Hepatology. 1999;29(1): 62-7.

6. $\operatorname{Rim} \mathrm{CH}$, Kim CY, Yang DS, Yoon WS. External beam radiation therapy to hepatocellular carcinoma involving inferior vena cava and/or right atrium: a meta-analysis and systemic review. Radiother Oncol. 2018;129(1):123-9.

7. Bruix J, Sherman M. Management of hepatocellular carcinoma. Hepatology. 2005;42(5):1208-36.

8. Miller $A B$, Hoogstraten $B$, Staquet $M$, Winkler A. Reporting results of cancer treatment. Cancer. 1981;47(1):207-14.

9. Hou JZ, Zeng ZC, Zhang JY, Fan J, Zhou J, Zeng MS. Influence of tumor thrombus location on the outcome of external-beam radiation therapy in advanced hepatocellular carcinoma with macrovascular invasion. Int J Radiat Oncol Biol Phys. 2012;84(2):362-8.

10. Zeng ZC, Fan J, Tang ZY, Zhou J, Qin LX, Wang JH, et al. A comparison of treatment combinations with and without radiotherapy for hepatocellular carcinoma with portal vein and/or inferior vena cava tumor thrombus. Int J Radiat Oncol Biol Phys. 2005;61(2):432-43.

11. Igaki H, Nakagawa K, Shiraishi K, Shiina S, Kokudo N, Terahara A, et al. Threedimensional conformal radiotherapy for hepatocellular carcinoma with inferior vena cava invasion. Jpn J Clin Oncol. 2008;38(6):438-44.

12. Koo JE, Kim JH, Lim YS, Park SJ, Won HJ, Sung KB, et al. Combination of transarterial chemoembolization and three-dimensional conformal radiotherapy for hepatocellular carcinoma with inferior vena cava tumor thrombus. Int J Radiat Oncol Biol Phys. 2010;78(1):180-7.

13. Xi M, Zhang L, Zhao L, Li QQ, Guo SP, Feng ZZ, et al. Effectiveness of stereotactic body radiotherapy for hepatocellular carcinoma with portal vein and/or inferior vena cava tumor thrombosis. PLoS One. 2013;8(5):e63864.

14. Duan F, Yu W, Wang Y, Liu FY, Song P, Wang ZJ, et al. Trans-arterial chemoembolization and external beam radiation therapy for treatment of hepatocellular carcinoma with a tumor thrombus in the inferior vena cava and right atrium. Cancer Imaging. 2015;15:7.

15. Komatsu S, Fukumoto T, Demizu Y, Miyawaki D, Terashima K, Niwa Y, et al. The effectiveness of particle radiotherapy for hepatocellular carcinoma associated with inferior vena cava tumor thrombus. J Gastroenterol. 2011; 46(7):913-20.

16. Kouloulias V, Mosa E, Georgakopoulos J, Platoni K, Brountzos I, Zygogianni A, et al. Three-dimensional conformal radiotherapy for hepatocellular carcinoma in patients unfit for resection, ablation, or chemotherapy: a retrospective study. ScientificWorldJournal. 2013;2013:780141.

Ready to submit your research? Choose BMC and benefit from:

- fast, convenient online submission

- thorough peer review by experienced researchers in your field

- rapid publication on acceptance

- support for research data, including large and complex data types

- gold Open Access which fosters wider collaboration and increased citations

- maximum visibility for your research: over $100 \mathrm{M}$ website views per year

At $\mathrm{BMC}$, research is always in progress.

Learn more biomedcentral.com/submissions 